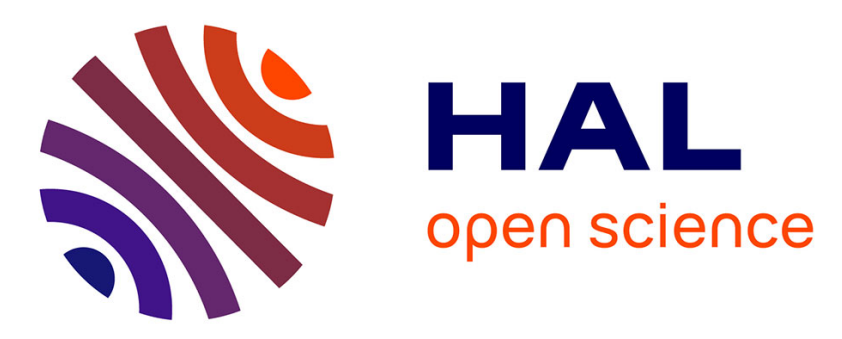

\title{
Justification of the cavity model in the numerical simulation of patch antennas by the method of matched asymptotic expansions
}

Abderrahmane Bendali, Abdelkader Makhlouf, Sébastien Tordeux

\section{- To cite this version:}

Abderrahmane Bendali, Abdelkader Makhlouf, Sébastien Tordeux. Justification of the cavity model in the numerical simulation of patch antennas by the method of matched asymptotic expansions. Multiscale Modeling and Simulation: A SIAM Interdisciplinary Journal, 2010, 8 (5), pp.1902-1922. 10.1137/090771041 . hal-00644409

\author{
HAL Id: hal-00644409 \\ https://hal.inria.fr/hal-00644409
}

Submitted on 13 Dec 2019

HAL is a multi-disciplinary open access archive for the deposit and dissemination of scientific research documents, whether they are published or not. The documents may come from teaching and research institutions in France or abroad, or from public or private research centers.
L'archive ouverte pluridisciplinaire HAL, est destinée au dépôt et à la diffusion de documents scientifiques de niveau recherche, publiés ou non, émanant des établissements d'enseignement et de recherche français ou étrangers, des laboratoires publics ou privés. 


\title{
JUSTIFICATION OF THE CAVITY MODEL IN THE NUMERICAL SIMULATION OF PATCH ANTENNAS BY THE METHOD OF MATCHED ASYMPTOTIC EXPANSIONS
}

\author{
ABDERRAHMANE BENDALI ${ }^{\dagger}$, ABDELKADER MAKHLOUF ${ }^{\dagger \S}$, AND SÉBASTIEN \\ TORDEUX ${ }^{\dagger}$
}

\begin{abstract}
The cavity model is a wide-spread empirical approach for the numerical simulation of patch antennas. An attempt to give a rigorous mathematical background for this way to proceed is presented. The justification is carried out in the framework of a two-dimensional representation of the underlying radiation problem. It is obtained by a suitable application of the method of matched asymptotic expansions. Furthermore, it is shown how to improve the cavity model by pushing the asymptotic expansion to the next order. A remarkable outcome of the asymptotic expansions is that they clearly confirm that the way to feed the antenna only determines the level of its radiation pattern but not its shape. Some numerical experiments are given to illustrate the theoretical developments.
\end{abstract}

Key words. Patch Antennas, Matched Asymptotic Expansions, Cavity Model

AMS subject classifications. 78A50, 78M35, 35J05

1. Introduction. Patch antennas are devices radiating or receiving electromagnetic waves that are used when a low-profile shape becomes an important requirement. They generally consist of a metallic strip placed at a small distance above a larger ground plane (see [1, 4]). Several approaches can be used for the development of analytical and numerical models predicting the functioning of these antennas (see, e.g., $[4])$. We focus here on the "full-waves" and the "cavity" models, which are among the most representative of these methods. The "full-waves" (or Direct Numerical Simulation (DNS)) approach generally consists of a direct discretization of the Maxwell equations, supplemented by some transmission and boundary conditions, by means of a boundary element method [11] (often called 'method of moments' in the electrical engineering literature) or by a finite element method (see, e.g., [7]). The main disadvantage of DNS is two-fold. Neither does it take into account the reduction of dimension in the cavity, the region between the patch and the ground plane in the terminology of patch antennas, nor does it consider the singularity near the edges of the patch. By contrast, the cavity model correctly handles these difficult behaviors. In addition it dramatically reduces the computational cost in the three-dimensional case, since, then, with the further assumption that the ground plane is infinite, the radiation pattern of the antenna can be determined with sufficient accuracy from a simple explicit analytical formula (cf. [1]). However, the way to obtain this model remains questionable and not suited to improvements - maybe to corrections - which could be necessary for this approach. This paper presents a tentative study for filling these gaps.

The full 3D problem seems to be mathematically out of reach, at least to the extent considered here. This study is thus limited to a $2 \mathrm{D}$ model of the actual electromagnetic radiation problem. Since a rectangular patch antenna does not radiate in TM polarization, we accordingly assume that the electromagnetic field is TE, or in

\footnotetext{
${ }_{\dagger}^{\dagger}$ Université de Toulouse, Institut Mathématique de Toulouse, Département de Génie Mathématique, INSA de Toulouse, 135 avenue de Rangueil, F-31077 Toulouse cedex 4 (FRANCE)

$\ddagger$ abendali@insa-toulouse.fr

$\S_{\text {makhlouf@insa-toulouse.fr }}$

Istordeux@insa-toulouse.fr
} 
other words, that the electric field is in the propagation plane. It is also worth mentioning that the cavity model brings back the determination of the field emitted by this kind of patch antenna to that of two parallel lines of constant magnetic currents placed above the ground plane [1]. The radiation of this setup can be expressed by an analytical formula in a close form (see, e.g., [1]). The TE assumption can be seen as a way to obtain the field emitted per-unit length of these lines in the E plane and hence correctly accounts for the field radiated by the patch antenna, at least in this plane. Additional assumptions will be introduced below to significantly simplify the mathematical framework while keeping the characteristic features of the field emitted by the antenna. We do not make the usual assumption that the ground plane is infinite. We thought it is important to show how dealing with a finite one can be obtained in a simple way by adding a correction to the infinite ground plane solution. We shall however give some numerical experiments below showing that, even with a relatively not too large ground plane, the model obtained by assuming that it is infinite, yields the radiation pattern with enough accuracy in the appropriate directions for the functioning of the antenna. We therefore assume that the patch is placed above a planar part of a larger finite metallic structure. Figure FIG. 1.1 sketches a plane view of the geometry.

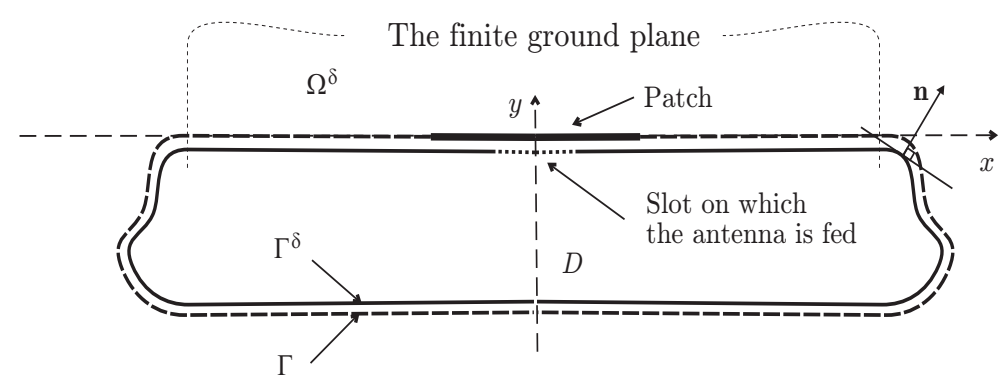

FIG. 1.1. Geometry of the radiating structure: $\Omega^{\delta}$ is the cracked domain obtained by excluding from the complement of the domain $D$ enclosed by the curve $\Gamma^{\delta}$ the segment $P$ representing the patch.

We do not consider geometries with singularities other than those located at the opening of the cavity. Such a consideration would lead to additional difficulties which are not specific to the concern of this study and which were dealt with elsewhere [3]. We hence assume that the metallic structure occupies a bounded domain $D$ enclosed in a sufficiently smooth curve $\Gamma^{\delta}$ which is furthermore a straight line on its part below the patch. To simplify the notation, we also assume that the part of $\Gamma^{\delta}$ which does not coincide with the ground plane $\{y=-\pi \delta\}$ lies in the half-plane $\{y<-\pi \delta\}$. The antenna is loaded through an electric field prescribed on a slot in the ground plane as indicated in FIG. 1.1. Some formulas occurring below take a simpler form if the distance separating the patch from the ground plane is set to $\pi \delta$, where $\delta>0$ is the small parameter leading this device to behave like a patch antenna. On this account, the geometrical representation of the patch is then the segment

$$
P:=\left\{(x, 0) \in \mathbb{R}^{2} ;|x| \leq L\right\}
$$

We denote by $\Omega^{\delta}$ the complement of $D \cup \Gamma^{\delta} \cup P$ (see FIG. 1.1). The boundary-value 
problem characterizing the emitted field can be stated as follows

$$
\left\{\begin{array}{l}
\Delta u^{\delta}+\kappa^{2} u^{\delta}=0 \text { in } \Omega^{\delta} \\
\partial_{\mathbf{n}} u^{\delta}=\pi \delta \chi_{a} \text { on } \Gamma^{\delta}, \partial_{y} u^{\delta}\left(x, 0^{ \pm}\right)=0 \text { for }|x|<L, \\
\lim _{r \rightarrow \infty} r^{1 / 2}\left(\partial_{r} u^{\delta}-i \kappa u^{\delta}\right)=0,
\end{array}\right.
$$

where $\mathbf{n}$ is the unit normal to $\Gamma^{\delta}$ pointing outwards to $D, \chi_{a}$ the characteristic function of the "slot" $\left\{(x,-\pi \delta) \in \mathbb{R}^{2} ;|x| \leq a\right\}, a<L$ the half-length of the latter, $r=\sqrt{x^{2}+y^{2}}$ and $\kappa>0$ is the wave number. Classical notation is used without further comment.

The most natural way to prescribe the electric field on the slot is to assume that it is equal there to a constant $E_{0}$. The corresponding field radiated by the antenna would then be $\left(E_{0} / \pi \delta\right) u^{\delta}$, where $u^{\delta}$ is the solution to (1.1). Choosing $E_{0}=\pi \delta$ is only a convenient way to write the asymptotic expansion.

If $n_{x}$ and $n_{y}$ stand for the respective components of $\mathbf{n}$ and $\delta$ is sufficiently small, the following set

$$
\Gamma:=\left\{(x, y) \in \mathbb{R}^{2} ; x=x^{\prime}+\pi \delta n_{x}, y=y^{\prime}+\pi \delta n_{y},\left(x^{\prime}, y^{\prime}\right) \in \Gamma^{\delta}\right\},
$$

is a curve parallel to $\Gamma^{\delta}$ containing $P$. It is also worth noting that $\mathbf{n}$ is a unit normal to $\Gamma$ as well. For an actual patch antenna problem, the domain between $\Gamma^{\delta}$ and $\Gamma$ is filled by a dielectric layer, the substrate in patch antenna terminology. Here we neglect the contrast between the substrate and the ambient medium. This contrast as well as discontinuities of the unit normal $\mathbf{n}$ to $\Gamma^{\delta}$ far enough from the cavity can be taken into account by adapting the techniques developed in [3] (see also [9] where this adaptation was carried out for a simplified version of the problem addressed here but involving a substrate with a contrast). These issues are not within the actual concerns of the present study and will not be discussed here. We also assume that the geometry is symmetric relatively to the $y$-axis. This hypothesis considerably simplifies the calculations while preserving the essential characteristics of the electromagnetic field.

The outline of the paper is as follows. In a first section, we show how the general principles leading to the cavity model can be used to get a heuristic approximate solution to problem (1.1). In a next section taking $\delta$ as a small parameter tending to 0 , we use the method of matched asymptotic expansions to establish that the cavity model actually produces an approximate expression for the first-order expansion of $u^{\delta}$. We also show how a more accurate but also unfortunately much more elaborate model can be obtained by pushing the asymptotic expansion to the second order. The method of matched asymptotic expansions, as well as the conformal transform used for solving the related problems inside the boundary layer, are adaptations of those given in [10] for dealing with the scattering of an acoustic wave by a pair of semi-infinite barriers. However the second-order expansion requires another handling for the rules of matching as well as a sharper description of the singular behavior of $u^{\delta}$ near the edges of the patch. The final section is dedicated to some numerical experiments comparing the results which can be obtained through a DNS approach, the cavity with magnetic walls model, and the matched asymptotic expansions method respectively.

2. The cavity model. The cavity model is obtained by first assuming that the region between the patch and the ground plane

$$
\Omega_{\mathrm{c}}^{\delta}:=\left\{(x, y) \in \mathbb{R}^{2} ;|x|<L,-\pi \delta<y<0\right\}
$$


is bounded by electric conductors and by magnetic walls (see FIG. 2.1). This makes it possible to calculate an approximation to the field inside the cavity. The field emitted by the antenna is then obtained from its values on the side walls of the cavity which become available after the previous step. Actually, the approximate problem within the cavity is generally not solved in an exact way, but by means of a kind of spectral method based on a modal approximation by the dominant mode (cf. e.g., [1]). For the present case, the boundary-value problem to be solved is the following

$$
\left\{\begin{array}{l}
\Delta u_{\mathrm{c}}^{\mathrm{cm}}+\kappa^{2} u_{\mathrm{c}}^{\mathrm{cm}}=0 \text { in } \Omega_{\mathrm{c}}^{\delta}, \\
\partial_{y} u_{\mathrm{c}}^{\mathrm{cm}}\left(x, 0^{-}\right)=0 \text { and } \partial_{y} u_{\mathrm{c}}^{\mathrm{cm}}\left(x,-\delta \pi^{+}\right)=\delta \pi \chi_{a} \text { for }|x|<L . \\
u_{\mathrm{c}}^{\mathrm{cm}}( \pm L, y)=0 \text { for }-\pi \delta<y<0,
\end{array}\right.
$$

where superscript "cm" is put to distinguish between the approximation heuristically obtained from the cavity model and those, given below, which are built by using an asymptotic expansion of $u^{\delta}$. In the same way, subscript "c" is used to refer to the expression for the field inside the cavity. Below, we shall also denote by $u^{\mathrm{cm}}$ the approximation of the field radiated by the antenna related to the cavity model. The approximate solution to $(2.2)$, obtained in terms of the dominant mode, is given by

$$
u_{\mathrm{c}}^{\mathrm{cm}}(x, y) \approx \frac{4}{\pi\left(\kappa^{2}-\pi^{2} / 4 L^{2}\right)} \sin \left(\frac{\pi a}{2 L}\right) \cos \left(\frac{\pi}{2 L} x\right)
$$

It is worth noting that this approximation can also be seen as a way to perform a dimension reduction of the solution inside the cavity. Since $u_{\mathrm{c}}^{\mathrm{cm}}$ does not depend on $y$, we simply write

$$
u_{\mathrm{c}}^{\mathrm{cm}}(x, y)=u_{\mathrm{c}}^{\mathrm{cm}}(x)
$$

Observe that $u_{\mathrm{c}}^{\mathrm{cm}}$ cannot be defined for the wave number

$$
\kappa=\pi / 2 L \text {. }
$$

This corresponds in fact to the first resonance frequency of the antenna. The reason of this flaw is that we have neglected the contrast of the substrate. Otherwise assuming an effective loss tangent for the material filling the domain limited by $\Gamma^{\delta}$ and $\Gamma$ would lead to a wave number $\kappa_{\mathrm{c}}$ in the cavity with a positive imaginary part. Of course this would prevent condition (2.5) from being satisfied.

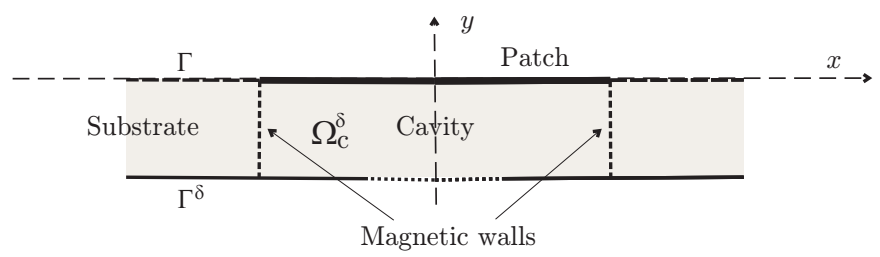

FIG. 2.1. The cavity zone: $\Omega_{c}^{\delta}$ is the domain between the patch and the ground plane.

The radiation through the side walls can then be obtained in the following way. A basic step is to consider this radiation for the case of an infinite ground plane, as this was mentioned above. We easily get that the corresponding field, denoted $u_{\infty}^{\mathrm{cm}}$, satisfies the following conditions

$$
\left\{\begin{array}{l}
\Delta u_{\infty}^{\mathrm{cm}}+\kappa^{2} u_{\infty}^{\mathrm{cm}}=0 \text { for } \Omega_{\infty}^{\delta} \\
\partial_{y} u_{\infty}^{\mathrm{cm}}\left(x, 0^{+}\right)=0 \text { for }|x|<L, \partial_{y} u_{\infty}^{\mathrm{cm}}\left(x,-\pi \delta^{+}\right)=0 \text { for }|x|>L \\
u_{\infty}^{\mathrm{cm}}( \pm L, y)=0, \partial_{x} u_{\infty}^{\mathrm{cm}}( \pm L, y)= \pm \partial_{x} u_{\mathrm{c}}^{\mathrm{cm}}(L), \text { for }-\pi \delta<y<0 \\
\lim _{|r| \rightarrow \infty}|r|^{1 / 2}\left(\partial_{r} u_{\infty}^{\mathrm{cm}}-i \kappa u_{\infty}^{\mathrm{cm}}\right)=0
\end{array}\right.
$$




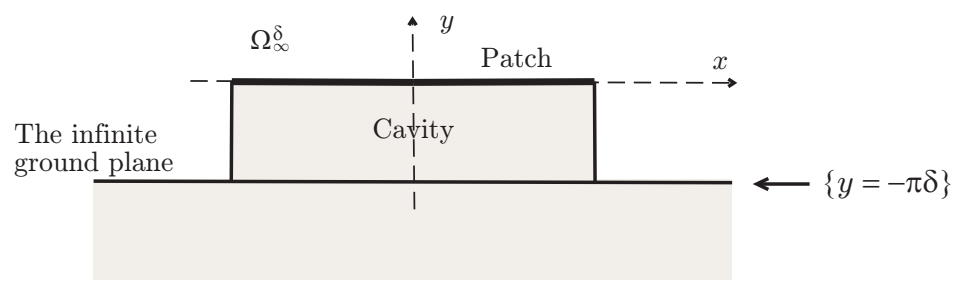

FIG. 2.2. Domain $\Omega_{\infty}^{\delta}$.

where $\Omega_{\infty}^{\delta}$ is the complement of the cavity relatively to the half-plane $\{y>-\pi \delta\}$ (in figure FIG. 2.2, $\Omega_{\infty}^{\delta}$ is represented as the part of the plane not in grey).

It is worth mentioning that the approximations of $u_{\infty}^{\mathrm{cm}}$ which are given below are the same, or at least of the same order, as those established in the engineering literature [1] by a more empirical approach based on the determination of the field radiated by two lines of equivalent magnetic currents placed above a ground plane.

The Dirichlet and Neumann conditions cannot be prescribed in general on the same part of the boundary. This illustrates the difficulty to get a direct mathematical justification of the cavity model. However, we can use the method of images to obtain an approximate solution assuming that the Dirichlet and the Neumann data are reasonably close to the actual ones. The procedure consists in writing an integral representation of

$$
\widetilde{u_{\infty}^{\mathrm{cm}}}(x, y)=u_{\infty}^{\mathrm{cm}}(x,|y+\pi \delta|) .
$$

This function is actually the extension by parity, relatively to the infinite ground plane $\{y=-\pi \delta\}$, of $u_{\infty}^{\mathrm{cm}}$. It is defined outside the domain

$$
\widetilde{\Omega_{\mathrm{c}}^{\delta}}:=\left\{(x, y) \in \mathbb{R}^{2} ;|x|<L,-2 \pi \delta<y<0\right\}
$$

obtained by extending symmetrically the cavity. According to the boundary-values of $\widetilde{u_{\infty}^{\mathrm{cm}}}$ and to the radiation condition satisfied by $u_{\infty}^{\mathrm{cm}}$, this integral representation is given by

$$
\begin{aligned}
\widetilde{u_{\infty}^{\mathrm{cm}}}(x, y) & =-\int_{-L}^{+L}\left(\partial_{y^{\prime}} G\left(x, y, x^{\prime}, 0\right)-\partial_{y^{\prime}} G\left(x, y, x^{\prime},-2 \pi \delta\right)\right) u_{\infty}^{\mathrm{cm}}\left(x^{\prime}, 0^{+}\right) d x^{\prime} \\
& -\int_{-2 \pi \delta}^{0} \partial_{x} u_{\mathrm{c}}^{\mathrm{cm}}(L)\left(G\left(x, y,-L, y^{\prime}\right)+G\left(x, y, L, y^{\prime}\right)\right) d y^{\prime}
\end{aligned}
$$

where $G\left(x, y, x^{\prime}, y^{\prime}\right)$ is the Green kernel giving the outgoing solutions to the Helmholtz equation

$$
G\left(x, y, x^{\prime}, y^{\prime}\right)=\frac{i}{4} H_{0}^{(1)}\left(\kappa \sqrt{\left(x-x^{\prime}\right)^{2}+\left(y-y^{\prime}\right)^{2}}\right)
$$

the function $H_{0}^{(1)}$ being the Hankel function of the first kind (see, e.g., $[5,6,2,12]$ ). For $(x, y)$ far enough from the cavity, the above representation can be approximated by an expression which does not involve the unknown boundary-value $u_{\infty}^{\mathrm{cm}}\left(x^{\prime}, 0^{+}\right)$for $\left|x^{\prime}\right|<L$

$$
\widetilde{u_{\infty}^{\mathrm{cm}}}(x, y) \approx \frac{\pi \delta}{2 i} \partial_{x} u_{\mathrm{c}}^{\mathrm{cm}}(L) H_{0}(x, y)
$$




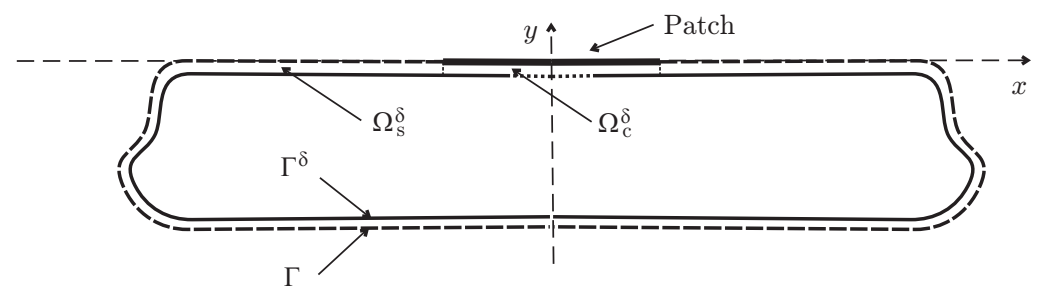

FIG. 3.1. The cavity $\Omega_{c}^{\delta}$ and the fictitious zone $\Omega_{s}^{\delta}$

with

$$
\left\{\begin{array}{l}
H_{0}^{+}(x, y)=H_{0}^{(1)}\left(\kappa \sqrt{(x-L)^{2}+y^{2}}\right), H_{0}^{-}(x, y)=H_{0}^{+}(-x, y), \\
H_{0}=H_{0}^{+}+H_{0}^{-}
\end{array}\right.
$$

Actually, the approximation (2.11) for the sum of the integrals in (2.9) represents a solution related to an infinite ground plane located at $\{y=0\}$. Using a midpoint approximation for the second integral in (2.9) would correspond to a solution obtained by considering the actual ground plane $\{y=-\pi \delta\}$. However, proceeding as it is done here will enable us to make a direct comparison of the cavity model with the first order asymptotic expansion of $u^{\delta}$. As a consequence, we correct the Neumann condition on the part of the curve $\Gamma$ which is lying in the half-plane $\{y<0\}$ even if this correction appears to be more straightforward on $\Gamma^{\delta}$. Hence, defining $g_{0}$ on $\Gamma$ by $g_{0}(x, y)=-\partial_{\mathbf{n}} H_{0}(x, y)$, if $y<0$ and $g_{0}(x, y)=0$ elsewhere, $\Omega$ as the unbounded domain limited by $\Gamma$, and $W_{0}$ as the solution to the Neumann problem

$$
\left\{\begin{array}{l}
\Delta W_{0}+\kappa^{2} W_{0}=0 \text { in } \Omega \\
\partial_{\mathbf{n}} W_{0}=g_{0} \text { on } \Gamma \\
\lim _{|r| \rightarrow \infty}|r|^{1 / 2}\left(\partial_{r} W_{0}-i \kappa W_{0}\right)=0
\end{array}\right.
$$

we obtain an approximate value of the field radiated by the patch corresponding to the cavity model

$$
u^{\delta} \approx u^{\mathrm{cm}} \approx \frac{\pi \delta}{2 i} \partial_{x} u_{\mathrm{c}}^{\mathrm{cm}}(L)\left(H_{0}+W_{0}\right) .
$$

\section{The method of matched asymptotic expansions.}

3.1. General equations. The field inside the cavity $\Omega_{\mathrm{c}}^{\delta}$ has a behavior which is mainly one-dimensional on the whole. Its matching with the field outside the cavity, which is two-dimensional by nature, has to be done through two boundary layers respectively located around the point $(L, 0)$ and $(-L, 0)$. By symmetry, it will be enough to carry out this matching at the point $(L, 0)$.

Hence, two regions have to be distinguished to correctly describe the field: the interior of the cavity and its exterior. However, considering a fictitious zone $\Omega_{\mathrm{s}}^{\delta}$, exterior to $\Omega_{\mathrm{c}}^{\delta}$ and comprised between $\Gamma^{\delta}$ and $\Gamma$, is helpful for obtaining the asymptotic expansion (see Fig. 3.1).

The variations of the field inside the boundary layer regions are best described as functions of the fast variables, related below to the right side of the cavity,

$$
X=(x-L) / \delta, Y=y / \delta .
$$




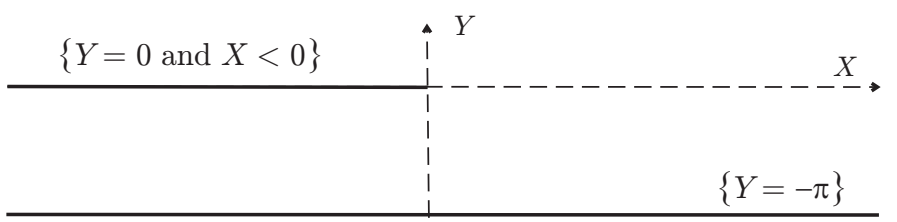

FIG. 3.2. The domain $\widehat{\Omega}$ used for the representation of the field inside the boundary layer zone near the right edge of the patch.

These fast variables run through the unbounded domain $\widehat{\Omega}$ obtained by excluding the semi-infinite line $\left\{(X, 0) \in \mathbb{R}^{2} ; X \leq 0\right\}$ from the half-plane $\{Y>-\pi\}$. This domain has therefore a straight boundary $\widehat{\Gamma}$ consisting of the previous semi-line and the line $\left\{(X,-\pi) \in \mathbb{R}^{2} ; X \in \mathbb{R}\right\}$ (see Fig. 3.2).

The expansion of $u^{\delta}$ can then be obtained in the above three regions as follows

$$
\begin{gathered}
u^{\delta}(x, y)=\sum_{n=0}^{N} \sum_{m=0}^{n} \delta^{n} \ln ^{m} \delta u^{n, m}(x, y)+o\left(\delta^{N}\right) \text { for }(x, y) \in \Omega, \\
u^{\delta}(x, y)=\sum_{n=0}^{N} \sum_{m=0}^{n} \delta^{n} \ln ^{m} \delta u_{\mathrm{s}}^{n, m}(s, t)+o\left(\delta^{N}\right) \text { for }(x, y) \in \Omega_{\mathrm{s}}^{\delta}, \\
u^{\delta}(x, \delta Y)=\sum_{n=0}^{N} \sum_{m=0}^{n} \delta^{n} \ln ^{m} \delta u_{\mathrm{c}}^{n, m}(x, Y)+o\left(\delta^{N}\right) \text { for }(x, \delta Y) \in \Omega_{\mathrm{c}}^{\delta},
\end{gathered}
$$

where $(s, t)$ is linked to $(x, y)$ through the relation

$$
(x, y)=\left(x(s)+\delta t n_{x}, y(s)+\delta t n_{y}\right)
$$

$s$ being the unit-speed parameter of $\Gamma$ increasing in the clockwise direction such that $s=0$ for $(x, y)=(L, 0)$. It is implicitly assumed that $n_{x}$ and $n_{y}$ are the components of the normal at $(x(s), y(s))$. The terms $\delta^{n} \ln ^{m} \delta$ for $m>0$ are generated by the singular behavior of the field at the junctions between the cavity and its exterior. A last expansion, expressed in an appropriate scaling, is required for the description of the boundary layers. It is enough by symmetry to deal with the point $(L, 0)$ in the proximity of which $u^{\delta}$ is assumed to have the following expansion

$$
u^{\delta}(L+\delta X, \delta Y)=\sum_{n=0}^{N} \sum_{m=0}^{n} \delta^{n} \ln ^{m} \delta \Pi^{n, m}(X, Y)+o\left(\delta^{N}\right)
$$

This kind of matched asymptotic expansions, at first-order only (that is, for $N=$ 1 ), was considered in [10] to build simpler models for several scattering problems. It was extended for expansions at an arbitrary order $N$ in [8] but in the framework of a specific scattering problem. For the study carried out here, we limit ourselves to second-order expansions (that is, for $N=2$ ).

Before setting the equations satisfied by the coefficients of these expansions, we need to express the Laplacian in coordinates $s$ and $t$. This expression

$$
\Delta u^{\delta}=\frac{1}{1+\delta t / \mathcal{R}} \partial_{s} \frac{1}{1+\delta t / \mathcal{R}} \partial_{s} u^{\delta}+\frac{1}{\delta^{2}} \frac{1}{1+\delta t / \mathcal{R}} \partial_{t}(1+\delta t / \mathcal{R}) \partial_{t} u^{\delta}
$$

makes use of the (signed) radius of curvature $\mathcal{R}$ of $\Gamma$ which is defined by the following relation

$$
\partial_{s} \mathbf{n}=\boldsymbol{\tau} / \mathcal{R}
$$


where $\boldsymbol{\tau}$ is the unit vector tangent to $\Gamma$ obtained by a clockwise rotation of $\pi / 2$ of $\mathbf{n}$. We can then expand the Helmholtz equation in the following form

$$
\left\{\begin{aligned}
\delta^{2}(1+\delta t / \mathcal{R}) & \left(\Delta u^{\delta}+\kappa^{2} u^{\delta}\right)=\partial_{t}^{2} u^{\delta}+\delta \partial_{t}(t / \mathcal{R}) \partial_{t} u^{\delta} \\
& +\delta^{2}\left(\partial_{s}^{2}+\kappa^{2}\right) u^{\delta}-\delta^{3}\left(\partial_{s}(t / \mathcal{R}) \partial_{s}-\kappa^{2} t / \mathcal{R}\right) u^{\delta} \\
& +\sum_{n=4}^{N}(-1)^{n} \delta^{n} \partial_{s}(t / \mathcal{R})^{n-2} \partial_{s} u^{\delta}+o\left(\delta^{N}\right)=0 .
\end{aligned}\right.
$$

Plugging the above expansions into system (1.1), collecting terms in $\delta^{n} \ln ^{m} \delta$, and equating them to 0 result in the following system. The first set of equations corresponds to the zone $\Omega$ where the radiated wave is propagating

$$
\left\{\begin{array}{l}
\Delta u^{n, m}+\kappa^{2} u^{n, m}=0 \text { in } \Omega, \\
\partial_{y} u^{n, m}\left(x, 0^{+}\right)=0 \text { for }|x|<L, \\
\lim _{r \rightarrow \infty} r^{1 / 2}\left(\partial_{r} u^{n, m}-i \kappa u^{n, m}\right)=0 .
\end{array}\right.
$$

A second set is posed inside the substrate

$$
\left\{\begin{aligned}
& \partial_{t}^{2} u_{\mathrm{s}}^{n, m}+ \partial_{t}(t / \mathcal{R}) \partial_{t} u_{\mathrm{s}}^{n-1, m}+\left(\partial_{s}^{2}+\kappa^{2}\right) u_{\mathrm{s}}^{n-2, m} \\
&-\left(\partial_{s}(t / \mathcal{R}) \partial_{s}-\kappa^{2} t / R\right) u_{\mathrm{s}}^{n-3, m} \\
&+\sum_{\ell=4}^{n}(-1)^{\ell} \partial_{s}(t / \mathcal{R})^{\ell-2} \partial_{s} u_{\mathrm{s}}^{n-\ell, m}=0 \text { in } \Omega_{\mathrm{s}}^{\delta} \\
& \partial_{t} u_{\mathrm{s}}^{n, m}(s,-\pi)=0 \text { for } s \text { such that }(x(s), y(s)) \in \Gamma_{\mathrm{s}}
\end{aligned}\right.
$$

The third one is related to the cavity

$$
\left\{\begin{array}{l}
\partial_{Y}^{2} u_{\mathrm{c}}^{n, m}+\left(\partial_{x}^{2}+\kappa^{2}\right) u_{\mathrm{c}}^{n-2, m}=0 \text { in } \Omega_{\mathrm{c}}^{\delta} \\
\partial_{Y} u_{\mathrm{c}}^{n, m}\left(x, 0^{-}\right)=0 \\
\partial_{Y} u_{\mathrm{c}}^{n, m}(x,-\pi)=0,(n, m) \neq(2,0) \text { for }|x|<L \\
\partial_{Y} u_{\mathrm{c}}^{20}(x,-\pi)=\pi \chi_{a}(x)
\end{array}\right.
$$

The transition region between the cavity and its exterior is governed by the equations posed in the boundary layer in terms of the fast variables

$$
\begin{aligned}
& \left(\partial_{X}^{2}+\partial_{Y}^{2}\right) \Pi^{n, m}+\kappa^{2} \Pi^{n-2, m}=0 \text { in } \widehat{\Omega}, \\
& \partial_{Y} \Pi^{n, m}=0 \text { on } \widehat{\Gamma} .
\end{aligned}
$$

These sets are complemented by the transmission conditions

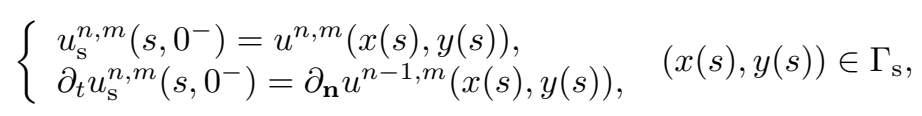

where $\Gamma_{\mathrm{s}}$ is the part of $\Gamma$ limiting $\Omega_{\mathrm{s}}^{\delta}$ (i.e., such that $\Gamma_{\mathrm{s}}$ and the patch $P$ constitute a non overlapping decomposition of $\Gamma$, see Fig. 1.1). In the above system, the equations are set for $0 \leq m \leq n$. Any term corresponding to a pair $(n, m)$ outside this range is equal to 0. By symmetry, it is also implicitly assumed that every function involved in the above system is an even function of the variable $x$.

3.2. The matching rules. It remains to set out the matching conditions linking the outer solutions depending on the slow variables $x$ or $(x, y)$ to the inner solutions depending on the fast variables $(X, Y)$. The matching rules given in [10] make it possible to determine the asymptotic expansion up to order 1 only. We had to develop a specific matching procedure to be able to describe the matching up to order 2 . At 
first, we remark that only $u^{n, m}$ and $u_{\mathrm{c}}^{n, m}$ have to be matched with $\Pi^{n, m}$. The close connection of $u_{\mathrm{s}}^{n, m}$ to $u^{n, m}$ insures its matching with $\Pi^{n, m}$. The matching procedure can hence be formulated as follows. Asymptotic expansion (3.2) is truncated at order $N$ in powers of $\delta$, and next expressed in terms of the fast variables yielding functions depending on $\delta$ and the fast variables $X$ and $Y$

$$
\begin{aligned}
& \left.\left(\sum_{n=0}^{N} \sum_{m=0}^{n} \delta^{n} \ln ^{m} \delta u^{n, m}(x, y)\right)\right|_{x=L+\delta X, y=\delta Y}=\sum_{n=0}^{N} \sum_{m=0}^{n} \delta^{n} \ln ^{m} \delta u^{n, m}(L+\delta X, \delta Y) \\
& \left.\left(\sum_{n=0}^{N} \sum_{m=0}^{n} \delta^{n} \ln ^{m} \delta u_{\mathrm{c}}^{n, m}(x, Y)\right)\right|_{x=L+\delta X}=\sum_{n=0}^{N} \sum_{m=0}^{n} \delta^{n} \ln ^{m} \delta u_{\mathrm{c}}^{n, m}(L+\delta X, Y) .
\end{aligned}
$$

We then expand the right-hand side of (3.15) and (3.16) as asymptotic series in $\delta^{p} \ln ^{q} \delta$ and remove any term negligible in comparison to $\delta^{N}$. The coefficients of these expansions yield functions $U_{N}^{n, m}(X, Y)$ and $U_{\mathrm{c} N}^{n, m}(X, Y)$ depending on the fast variables only

$$
\begin{aligned}
& \sum_{n=0}^{N} \sum_{m=0}^{n} \delta^{n} \ln ^{m} \delta u^{n, m}(L+\delta X, \delta Y)=\sum_{n=0}^{N} \sum_{m=0}^{n} \delta^{n} \ln ^{m} \delta U_{N}^{n, m}(X, Y)+o\left(\delta^{N}\right) \\
& \sum_{n=0}^{N} \sum_{m=0}^{n} \delta^{n} \ln ^{m} \delta u_{\mathrm{c}}^{n, m}(L+\delta X, Y)=\sum_{n=0}^{N} \sum_{m=0}^{n} \delta^{n} \ln ^{m} \delta U_{\mathrm{c} N}^{n, m}(X, Y)+o\left(\delta^{N}\right)
\end{aligned}
$$

The matching conditions are then directly expressed in terms of these coefficients

$$
\begin{gathered}
\Pi^{n, m}(X, Y)=U_{N}^{n, m}(X, Y)+\underset{R \rightarrow \infty}{o}\left(R^{n-N}\right) \\
\Pi^{n, m}(X, Y)=U_{\mathrm{c} N}^{n, m}(X, Y)+\underset{X \rightarrow-\infty}{o}\left(|X|^{n-N}\right)
\end{gathered}
$$

for $0 \leq m \leq n \leq N$ with $R:=\sqrt{X^{2}+Y^{2}}$.

Before going further in the determination of the asymptotic expansions, we observe that equations (3.11) and (3.12) are simple linear second order differential equations with respect to $t$ and $Y$ respectively. A simple calculation hence reduces the determination of each term of the asymptotic expansion of the field inside the cavity to that of a single coefficient $A^{n, m}$ since we can write

$$
\left\{\begin{array}{l}
u_{\mathrm{c}}^{0,0}(x)=A^{0,0} \cos (\kappa x)+\int_{0}^{x} \kappa^{-1} \sin \kappa(x-s) \chi_{a}(s) d s \\
u_{\mathrm{c}}^{1, m}(x)=A^{1, m} \cos (\kappa x), \text { for } m=0,1
\end{array}\right.
$$

Likewise integrating (3.11) in $t$ yields

$$
\begin{array}{r}
\partial_{t} u_{\mathrm{s}}^{n+1, m}(s, 0)+\int_{-\pi}^{0}\left(\partial_{s}^{2}+\kappa^{2}\right) u_{\mathrm{s}}^{n-1, m} d t+\int_{-\pi}^{0}\left(-\partial_{s}(t / \mathcal{R}) \partial_{s}+\kappa^{2} t / R\right) u_{\mathrm{s}}^{n-2, m} d t \\
+\sum_{\ell=4}^{n}(-1)^{\ell} \int_{-\pi}^{0} \partial_{s}(t / \mathcal{R})^{\ell-2} \partial_{s} u_{\mathrm{s}}^{n+1-\ell, m} d t=0 .
\end{array}
$$


In view of (3.14), we then obtain

$$
\begin{aligned}
\left.\partial_{\mathbf{n}} u^{n, m}\right|_{\Gamma_{\mathrm{s}}} & +\int_{-\pi}^{0}\left(\partial_{s}^{2}+\kappa^{2}\right) u_{\mathrm{s}}^{n-1, m} d t+\int_{-\pi}^{0}\left(-\partial_{s}(t / \mathcal{R}) \partial_{s}+\kappa^{2} t / \mathcal{R}\right) u_{\mathrm{s}}^{n-2, m} d t \\
& +\sum_{\ell=4}^{n}(-1)^{\ell} \int_{-\pi}^{0} \partial_{s}(t / \mathcal{R})^{\ell-2} \partial_{s} u_{\mathrm{s}}^{n+1-\ell, m} d t=0 .
\end{aligned}
$$

It is worth mentioning that our objective is only to obtain the asymptotic expansion of the far field emitted by the antenna, i.e., of $u^{n, m}$ for $0 \leq m \leq n \leq 2$. These terms are successively determined for $N=0,1$ and 2. Obtaining $u^{n, m}$ makes use only of cavity terms $u_{\mathrm{c}}^{\ell, m}$ at previous orders $\ell<n$. Therefore, the calculation of $u_{\mathrm{c}}^{n, m}$ is viewed as an initial step towards the determination of $u^{n+1, m}$.

3.3. The asymptotic expansion at order 0 . We begin by defining $u^{0,0}$. Making use of (3.10) and (3.22), we get that $u^{0,0}$ satisfies

$$
\left\{\begin{array}{l}
\Delta u^{0,0}+\kappa^{2} u^{0,0}=0 \text { in } \Omega \\
\partial_{\mathbf{n}} u^{0,0}(x, y)=0 \text { for }(x, y) \in \Gamma \text { and }(x, y) \neq( \pm L, 0) \\
\lim _{r \rightarrow \infty} r^{1 / 2}\left(\partial_{r} u^{0,0}-i \kappa u^{0,0}\right)=0
\end{array}\right.
$$

This is a non standard boundary-value problem since the behavior of $u^{0,0}$ near the points $( \pm L, 0)$ is not yet fixed, but will be obtained through condition (3.19). A straightforward adaptation of the case of an half-plane [8] shows that $u^{0,0}$ can be expanded as follows

$$
u^{0,0}=\sum_{\ell=0}^{+\infty} C_{\ell}^{0,0}\left(H_{\ell}+W_{\ell}\right)
$$

in modes $H_{\ell}+W_{\ell}$, which are a generalization of mode $H_{0}+W_{0}$ defined in (2.12), (2.13), and (2.14), and which are given by

$$
\begin{gathered}
\left\{\begin{array}{l}
H_{\ell}^{+}(x, y):=H_{\ell}^{(1)}(\kappa \varrho) \cos \ell \varphi, H_{\ell}^{-}(x, y)=H_{\ell}^{+}(-x, y), \\
H_{\ell}:=H_{\ell}^{+}+H_{\ell}^{-}
\end{array}\right. \\
g_{\ell}(x, y):=\left\{\begin{array}{l}
0, \text { if } y=0, \\
-\partial_{\mathbf{n}} H_{\ell}(x, y), \text { if } y<0,
\end{array}\right. \\
\left\{\begin{array}{l}
\Delta W_{\ell}+\kappa^{2} W_{\ell}=0 \text { in } \Omega, \\
\partial_{\mathbf{n}} W_{\ell}=g_{\ell} \text { on } \Gamma \\
\lim _{r \rightarrow \infty} r^{1 / 2}\left(\partial_{r} W_{\ell}-i \kappa W_{\ell}\right)=0 .
\end{array}\right.
\end{gathered}
$$

Here, and in all the sequel, $\varrho$ and $\varphi$ designate the polar coordinates centered at $(L, 0)$. As usual, $H_{\ell}^{(1)}$ is the first kind Hankel function of non-negative integer order $\ell$. The determination of $u^{0,0}$ is then reduced to that of the complex coefficients $C_{\ell}^{0,0}$.

It is important then to remark that the asymptotic behavior of Bessel's functions for small values of the argument (cf., e.g., [14]) gives that

$$
\left(H_{m}+W_{m}\right)(\delta X, \delta Y)= \begin{cases}\mathcal{O}(\ln \delta), & \text { for } m=0 \\ \mathcal{O}\left(\delta^{-m}\right), & \text { for } m \geq 1\end{cases}
$$


As a result, if $C_{\ell}^{0,0} \neq 0$ for $\ell \geq 1$, the expansion of $u^{0,0}(\delta X, \delta Y)$ in $\delta$ will involve terms in $\delta^{-\ell}$ which cannot be matched with the coefficient $\Pi^{0,0}(X, Y)$ since it is a constant relatively to $\delta$. In the same way, if $C_{0}^{0,0} \neq 0, u^{0,0}(\delta X, \delta Y)$ will contain a term in $\ln \delta$ yielding an expression which is not bounded in $\delta$. This actually means that the matching rule expressed by condition (3.19) cannot be satisfied unless

$$
u^{0,0}=0 .
$$

Making use of (3.11) and (3.14) one immediately deduces that

$$
u_{\mathrm{s}}^{0,0}=0 .
$$

3.4. The asymptotic expansion at order 1 . We first determine $\Pi^{0,0}$. To do this, we use the following adaptation [9] of the conformal transform considered by McIver and Rawlins [10]

$$
X+i Y=1-\omega(X, Y)+\ln \omega(X, Y)
$$

which defines a one-to-one mapping between $\widehat{\Omega}$ and the lower half of the complex plane

$$
\{\omega \in \mathbb{C} ; \Im m(\omega)<0\} .
$$

It makes use of the principal branch of the logarithm which corresponds to a cut along the negative real axis. In view of the following elementary estimate

$$
\frac{e^{X-1} e^{i Y}}{\omega(X, Y)}=1+\underset{|\omega| \rightarrow 0}{\mathcal{O}}(\omega)
$$

which is a direct consequence of (3.31), it can be easily established (see $[10,9])$ that the only solution to

$$
\left\{\begin{array}{l}
\Delta_{X Y} \Pi=0 \\
\partial_{Y} \Pi=0 \text { on } \widehat{\Gamma},
\end{array}\right.
$$

growing at most as a polynomial in $X$ when $X \rightarrow-\infty$ for $-\pi<Y<0$, is in the form

$$
\Pi(X, Y)=a \Re e \ln \omega(X, Y)+\sum_{\ell=0}^{\infty} b_{\ell} \Re e \omega^{\ell}(X, Y) .
$$

Elementary estimates [9] yield

$$
\begin{aligned}
& \Re e \ln \omega(X, Y)=\ln R+\underset{R \rightarrow \infty}{o}(1) \\
& \Re e \omega^{n}(X, Y)=(-1)^{n} R^{n} \cos (n \Theta)+o\left(R^{n}\right) \text { for } n=0,1, \ldots \\
& \Re e \ln \omega(X, Y)=X-1+\underset{X \rightarrow-\infty}{o}\left(|X|^{-\infty}\right), \text { for }-\pi<Y<0 \\
& \Re e \omega^{n}(X, Y)=\underset{X \rightarrow-\infty}{o}\left(|X|^{-\infty}\right),
\end{aligned}
$$


where $R=\sqrt{X^{2}+Y^{2}}$ and $\Theta$ are the polar coordinates associated to $(X, Y)$ and $\underset{X \rightarrow-\infty}{o}\left(|X|^{-\infty}\right)$ indicates a function decreasing toward 0 faster than any negative power of $X$. Matching conditions (3.6) then imply that

$$
\Pi^{0,0}=0 .
$$

In turn, matching rule (3.20) gives the magnetic walls boundary condition

$$
u_{\mathrm{c}}^{0,0}( \pm L, 0)=0
$$

which was assumed a priori as a basic step in the above derivation of the cavity model. Inserting this into (3.21) directly gives the coefficient

$$
A^{0,0}=\frac{1}{\kappa^{2}}\left(1-\frac{\cos \kappa(L-a)}{\cos \kappa L}\right)
$$

which remains to be determined in the expression of $u_{\mathrm{c}}^{0,0}$ if $\kappa$ does not correspond to an eigenvalue of the two-points Sturm-Liouville problem, associated to

$$
\left\{\begin{array}{l}
\partial_{x}^{2} u_{\mathrm{c}}^{0,0}(x)+\kappa^{2} u_{\mathrm{c}}^{0,0}(x)=\chi_{a} \text { for }|x|<L \\
u_{\mathrm{c}}^{0,0}(L)=u_{\mathrm{c}}^{0,0}(-L)=0
\end{array}\right.
$$

that is, if $\kappa \neq \kappa_{\ell}$, with $\kappa_{\ell}=(2 \ell+1) \pi / 2 L(\ell=0,1,2, \ldots)$.

Proceeding as for $u^{0,0}$, we readily get that $u^{1,1}=0$ and that $u^{1,0}$ is expressed in terms of two complex constants $C_{0}^{1,0}$ and $C_{1}^{1,0}$ not yet determined

$$
u^{1,0}=C_{0}^{1,0}\left(H_{0}+W_{0}\right)+C_{1}^{1,0}\left(H_{1}+W_{1}\right) .
$$

Using the following asymptotic expansions of respectively $H_{0}^{(1)}(\delta \kappa R)$ and $H_{1}^{(1)}(\delta \kappa R)$ for $\delta \rightarrow 0$ (cf., e.g., [14])

$$
\begin{aligned}
& H_{0}^{(1)}(\delta k R)=\frac{2 i}{\pi} \ln \delta+\frac{2 i}{\pi} \ln R+\gamma_{\kappa}+\mathcal{O}\left(\delta^{2} \ln \delta\right) \\
& H_{1}^{(1)}(\delta k R)=-\frac{2 i}{\pi} \frac{1}{\kappa R} \delta^{-1}+\mathcal{O}(\delta \ln \delta)
\end{aligned}
$$

with

$$
\gamma_{\kappa}=1+\frac{2 i}{\pi}\left(\gamma+\ln \frac{\kappa}{2}\right)
$$

where $\gamma$ is Euler's constant, we readily get

$$
\begin{gathered}
U_{1}^{0,0}(X, Y)=C_{1}^{1,0}, \\
U_{1}^{1,0}(X, Y)=C_{0}^{1,0} \frac{2 i}{\pi} \ln R+C_{0}^{1,0}\left(\gamma_{\kappa}+\left(H_{0}^{-}+W_{0}\right)(L, 0)\right), \\
U_{1}^{1,1}(X, Y)=C_{0}^{10} \frac{2 i}{\pi} .
\end{gathered}
$$

We then make use of matching rules (3.19) to deduce that

$$
C_{1}^{1,0}=0
$$


and that

$$
\begin{aligned}
& \Pi^{1,0}(X, Y)=C_{0}^{1,0} \frac{2 i}{\pi} \ln R+C_{0}^{1,0}\left(\gamma_{\kappa}+\left(H_{0}^{-}+W_{0}\right)(L, 0)\right)+o(1), \\
& \Pi^{1,1}(X, Y)=C_{0}^{10} \frac{2 i}{\pi}+o(1) .
\end{aligned}
$$

Now, since both $\Pi^{1,0}$ and $\Pi^{1,1}$ satisfy (3.34), taking into account (3.35), (3.36), and (3.37), we get

$$
\begin{aligned}
& \Pi^{1,0}(X, Y)=C_{0}^{10} \frac{2 i}{\pi} \Re e \ln \omega(X, Y)+C_{0}^{10}\left(\gamma_{\kappa}+\left(H_{0}^{-}+W_{0}\right)(L, 0)\right), \\
& \Pi^{1,1}(X, Y)=C_{0}^{10} \frac{2 i}{\pi} .
\end{aligned}
$$

Finally, noting that $u_{\mathrm{c}}^{n, m}(0 \leq m \leq n \leq 1)$ does not depend on $Y$, we can write

$$
\begin{aligned}
& u_{\mathrm{c}}^{0,0}(L+\delta X)+\delta u_{\mathrm{c}}^{1,0}(L+\delta X)+\delta \ln \delta u_{\mathrm{c}}^{1,1}(L+\delta X)= \\
& \delta\left(\partial_{x} u_{\mathrm{c}}^{0,0}(L) X+u_{\mathrm{c}}^{1,0}(L)\right)+\delta \ln \delta u_{\mathrm{c}}^{1,1}(L)+\underset{X \rightarrow-\infty}{o}(1) .
\end{aligned}
$$

According to (3.38), one gets the following behavior of $\Pi^{1,0}$

$$
\Pi^{1,0}(X, Y)=\frac{2 i}{\pi} C_{0}^{1,0} X+C_{0}^{1,0}\left(\gamma_{\kappa}-2 i / \pi+\left(H_{0}^{-}+W_{0}\right)(L, 0)\right)+\underset{X \rightarrow-\infty}{o}(1)
$$

for $-\pi<Y<0$. From (3.55), we first have that

$$
U_{\mathrm{c} 1}^{0,0}(X, Y)=0, U_{\mathrm{c} 1}^{1,0}(X, Y)=\partial_{x} u_{\mathrm{c}}^{0,0}(L) X+u_{\mathrm{c}}^{1,0}(L), U_{\mathrm{c} 1}^{1,1}(X, Y)=u_{\mathrm{c}}^{1,1}(L) .
$$

In the same way, making use of matching rule (3.20), we obtain the last coefficient involved in the first-order asymptotic approximation of $u^{\delta}$

$$
C_{0}^{1,0}=\frac{\pi}{2 i} \partial_{x} u_{\mathrm{c}}^{0,0}(L)
$$

We can thus construct an approximate solution to (1.1) in terms of the following first-order asymptotic expansion

$$
u^{\delta}=\frac{\pi \delta}{2 i} \partial_{x} u_{\mathrm{c}}^{0,0}(L)\left(H_{0}+W_{0}\right)+o(\delta),
$$

which differs from the one corresponding to the cavity model (2.14) only by the multiplicative factor $\partial_{x} u_{\mathrm{c}}^{0,0}(L)$. A further inspection shows that, contrary to $\partial_{x} u_{\mathrm{c}}^{0,0}(L)$ which was calculated by solving the two-points problem $(3.42)$ exactly, $\partial_{x} u_{\mathrm{c}}^{\mathrm{cm}}(L)$ was obtained from an approximate solution to this problem built by keeping the top-order term only of the following modal expansion

$$
\left\{\begin{array}{l}
u_{\mathrm{c}}^{0,0}(x)=a_{0} \cos \left(\frac{\pi}{2 L} x\right)+a_{1} \cos \left(\frac{3 \pi}{2 L} x\right)+\cdots \\
u_{\mathrm{c}}^{\mathrm{cm}}(x)=a_{0} \cos \left(\frac{\pi}{2 L} x\right)
\end{array}\right.
$$

expressed in terms of the eigenfunctions of the underlying Sturm-Liouville problem.

Proceeding as for $u_{\mathrm{s}}^{0,0}$ we directly obtain $u_{\mathrm{s}}^{1,1}=0$ and

$$
u_{\mathrm{s}}^{1,0}(s, t)=u^{1,0}(x(s), y(s)) \text { for }(x(s), y(s)) \in \Gamma_{\mathrm{s}} .
$$


3.5. The asymptotic expansion at order 2. The initial step is to use the matching conditions (3.20), (3.54), (3.55) and (3.56) to determine the second term of the asymptotic expansion of the field inside the cavity:

$$
\begin{aligned}
& u_{\mathrm{c}}^{1,0}(L)=\frac{\pi}{2 i} \partial_{x} u_{\mathrm{c}}^{0,0}(L)\left(\gamma_{\kappa}-\frac{2 i}{\pi}+\left(H_{0}^{-}+W_{0}\right)(\kappa L, 0)\right) \\
& u_{\mathrm{c}}^{1,1}(L)=\partial_{x} u_{\mathrm{c}}^{00}(L) .
\end{aligned}
$$

Then, proceeding as for $u^{0,0}$ and $u^{1,1}$, we can assert that

$$
u^{2,2}=0 .
$$

Similarly to $u^{1,0}$ and $u^{1,1}$, we find that

$$
u^{2,1}=C_{0}^{2,1}\left(H_{0}+W_{0}\right)+C_{1}^{2,1}\left(H_{1}+W_{1}\right) .
$$

The determination of $u^{2,0}$ is a more involved task. Due to (3.22) and (3.61), this term satisfies a nonhomogeneous Neumann boundary condition with data behaving in a highly singular way near the points $( \pm L, 0)$

$$
\partial_{n} u^{2,0}=Y_{\Gamma_{s}} \partial_{x} u_{\mathrm{c}}^{0,0}(L) \frac{i \pi^{2}}{2}\left(\partial_{s}^{2}+\kappa^{2}\right)\left(H_{0}+W_{0}\right) \text { on } \Gamma \backslash\{( \pm L, 0)\} .
$$

The function $Y_{\Gamma_{\mathrm{s}}}$ is equal to 1 on $\Gamma_{\mathrm{s}}$ and 0 on $P$. Makhlouf [9] has developed a systematic procedure to construct solutions to the Helmholtz equation for this kind of singular Neumann data. Here, it yields the existence of a function $w^{2,0}$ solution to the same boundary-value problem than $u^{2,0}$ in the form

$$
w^{2,0}=\partial_{x} u_{\mathrm{c}}^{0,0}(L)\left(\chi s^{2,0}+r^{2,0}\right)
$$

with $\chi$ a suitable cut-off function equal to 1 near the points $( \pm L, 0)$. The function $s^{2,0}$ is an even function relatively to the $y$-axis, incorporating the singularity of $u^{2,0}$ resulting from the singular behavior of the Neumann data, which has the following expression near the point $(L, 0)$

$$
s^{2,0}(\varrho, \varphi)=-\left(\frac{\ln \varrho \cos \varphi-(\pi-\varphi) \sin \varphi}{\varrho}+\frac{\kappa^{2}}{2} \varrho \ln \varrho(\pi-\varphi) \sin \varphi\right)
$$

and $r^{2,0}$ a solution to the following boundary-value problem

$$
\left\{\begin{array}{l}
\Delta r^{2,0}+\kappa^{2} r^{2,0}=-f \text { in } \Omega \\
\partial_{\mathbf{n}} r^{2,0}=g \text { on } \Gamma \\
\lim _{r \rightarrow \infty} r^{1 / 2}\left(\partial_{r} r^{2,0}-i \kappa r^{2,0}\right)=0
\end{array}\right.
$$

with continuous data $f$ and $g$. Function $f$, of bounded support located in the halfplane $\{y>0\}$, is explicitly expressed in terms of $\chi, s^{20}$ and the Hankel function $H_{0}^{1}$. In particular, due to the fact that $f$ is obtained by truncating a function incorporating the most singular part of $u^{2,0}$ by means of a suitable cut-off function in a neighborhood of $( \pm L, 0)$ and that the geometry is straight in the proximity of these points, the support of $f$ can be chosen to be a rectangle, located in the half-plane $\{y>0\}$, having one side on $\Gamma$. Finally, proceeding in the same way as for $u^{1,0}$, the determination of $u^{2,0}-w^{2,0}$ is brought to that of two constants such that

$$
u^{2,0}=w^{2,0}+C_{0}^{2,0}\left(H_{0}+W_{0}\right)+C_{1}^{2,0}\left(H_{1}+W_{1}\right) .
$$


Straightforward but laborious calculations yield the second-order expansion similarly as for the first-order

$$
\begin{aligned}
C_{0}^{2,0} & =\frac{\pi^{2}}{4} \partial_{x} u_{\mathrm{c}}^{00}(L)\left(\begin{array}{c}
\left(\gamma_{\kappa}-2 i / \pi+\left(H_{0}^{-}+W_{0}\right)(\kappa L, 0)\right) \kappa \tan \kappa L+ \\
\partial_{x}\left(H_{0}^{-}+W_{0}\right)(\kappa L, 0)
\end{array}\right), \\
C_{1}^{2,0} & =\kappa \frac{\pi}{2 i} \partial_{x} u_{\mathrm{c}}^{0,0}(L), \\
C_{0}^{2,1} & =-\kappa \frac{\pi}{2 i} \tan \kappa L \partial_{x} u_{\mathrm{c}}^{0,0}(L), \\
C_{1}^{2,1} & =-\kappa \frac{\pi}{2 i} \partial_{x} u_{\mathrm{c}}^{0,0}(L) .
\end{aligned}
$$

It is worth mentioning that the matching conditions now require some identifications of terms linked to the behavior of $\Pi^{1,0}$ as $R \rightarrow \infty$. These identifications do not determine unknown coefficients but have to be seen as compatibility conditions satisfied by already defined coefficients. These compatibility conditions must be satisfied; otherwise the asymptotic expansion cannot exist.

We are hence in a position to obtain a second-order approximation of the solution $u^{\delta}$ to problem (1.1) from the following expansion

$$
\begin{aligned}
u^{\delta}= & \frac{\pi \delta}{2 i} \partial_{x} u_{\mathrm{c}}^{00}(L)\left(H_{0}+W_{0}\right) \\
& +\delta^{2}\left(\begin{array}{c}
w^{20}+C_{0}^{20}\left(H_{0}+W_{0}\right)+C_{1}^{20}\left(H_{1}+W_{1}\right)+ \\
\ln \delta\left(C_{0}^{21}\left(H_{0}+W_{0}\right)+C_{1}^{21}\left(H_{1}+W_{1}\right)\right)
\end{array}\right)+\underset{\delta \rightarrow 0}{o}\left(\delta^{2}\right) .
\end{aligned}
$$

4. Numerical validation. Our objective in this section is to compare the radiation pattern of $u^{\delta}$ obtained by means of the above models, among them the solution which can be expressed by the explicit analytical formula

$$
u_{\infty}^{\mathrm{cm}}(x, y)=\frac{\pi \delta}{2 i} \partial_{x} u_{\mathrm{c}}^{\mathrm{cm}}(L) H_{0}(x, y)
$$

which, as indicated in (2.11), is derived from the cavity model by furthermore assuming that the ground plane is infinite. Clearly, the approximate expression for this solution is meaningful only in the half-plane $\{y>-\pi \delta\}$.

It is a well-known fact that each solution $u$ to the Helmholtz equation, with a right-hand side equal to 0 outside a circle of sufficiently large radius, satisfying the radiation condition, is characterized by its far-field pattern $a(\theta)$ defined by (cf., e.g., [13])

$$
u(r, \theta)=\frac{e^{i \kappa r}}{\sqrt{r}} a(\theta)+\underset{r \rightarrow \infty}{o}\left(r^{-3 / 2}\right),
$$

where $(r, \theta)$ are the polar coordinates of the plane. The radiation pattern of $u$ in $\mathrm{dB}$ is then defined as the following function in $\theta$

$$
10 \log _{10} 2 \pi|a(\theta)|^{2} .
$$

When furthermore the right-hand side of the Helmholtz equation is 0 in all the domain and $u$ is compelled to satisfy a Neumann boundary condition, the solution can be expressed through an unknown density on the boundary by means of a doublelayer potential. This density can be calculated by a boundary element method (BEM) 
enabling a direct computation of the far-field pattern $a(\theta)$ (cf., e.g., [2]). The far-field patterns of $u^{\delta}, W_{0}$ and $W_{1}$ are obtained in the same way.

Two procedures can be used to compute the far-field pattern of $r^{2,0}$. The first one is based on a coupling of a Finite Element Method (FEM) with a BEM. A cumbersome part of the FEM calculation can be avoided in this case by using a structured mesh of a rectangle, which contains the support of $f$ as indicated above. The second one does not make use of the FEM and builds the solution by correcting the following expression

$$
r_{\infty}^{2,0}(x, y)=\int_{\mathbb{R}^{2}} G\left(x, y, x^{\prime}, y^{\prime}\right) f\left(x^{\prime},\left|y^{\prime}\right|\right) d x^{\prime} d y^{\prime}
$$

corresponding to the case where the geometry is approximated by an infinite ground plane

$$
\left\{\begin{array}{l}
\Delta v^{2,0}+\kappa^{2} v^{2,0}=0 \text { in } \Omega \\
\partial_{\mathbf{n}} v^{2,0}=g-\partial_{\mathbf{n}} r_{\infty}^{2,0} \text { on } \Gamma \\
\lim _{r \rightarrow \infty} r^{1 / 2}\left(\partial_{r} v^{2,0}-i \kappa v^{2,0}\right)=0 \\
r^{2,0}=r_{\infty}^{2,0}+v^{2,0}
\end{array}\right.
$$

It is worth noting that due to the property $\partial_{y} r_{\infty}^{2,0}=0$ for $y=0$ the evaluation of $\partial_{\mathbf{n}} r_{\infty}^{2,0}$ has to be done only for $y<0$, that is, outside the support of $f$. The function $\partial_{\mathbf{n}} r_{\infty}^{2,0}$ thus appears as a boundary integral of a non singular function which can be efficiently obtained by numerical quadratures only. The results provided by each of these two procedures are almost identical.

From the asymptotic expression of Bessel function $H_{0}^{(1)}(z)$ for $|z| \rightarrow \infty$ (cf., e.g., $[14]$ ), we readily get that the far-field pattern corresponding to $u_{\infty}^{\mathrm{cm}}$ is obtained by means of the analytical formula

$$
a(\theta)=-\delta \sqrt{2 i \pi} \partial_{x} u_{\mathrm{c}}^{\mathrm{cm}}(L) \cos (\kappa L \cos \theta) .
$$

Obviously no method based on a numerical solving of a boundary-value problem can outperform the simplicity of this approach in terms of CPU time within its domain of validity.

We have tested the configuration depicted in FIG. 4.1. The lengths are reported in normalized units.

The mesh size is fixed by means of the positive integer parameter dens which gives the number of points by wavelength everywhere except in the proximity of the edges of the patch and in the opening of the cavity (magnetic walls). We use a log scale near these points to handle the singularity of the field there. Moreover in these zones, we augment the parameter dens by a factor 5 .

Several tests have been carried out for various values of $\kappa$. The results were all similar provided $\kappa$ remains far enough from $\kappa_{\text {res }}=\pi / 0.79 \approx 1.27 \pi$ which corresponds to the first resonance of the patch. For these values of $\kappa$, the radiation patterns obtained either by the DNS or by any of the two asymptotic expansions are quite different. This confirms that these cases are outside the domain of validity of the considered models as predicted by the theoretical study. Since a patch antenna is generally used for a frequency at a small fraction below its resonance frequency, all the results presented below have been obtained with $\kappa=\pi$. Similarly, since the distance separating the patch from the ground plane is generally $1 / 100$ th the width of the former, we have taken $\delta=2 L / 100 \pi$. 


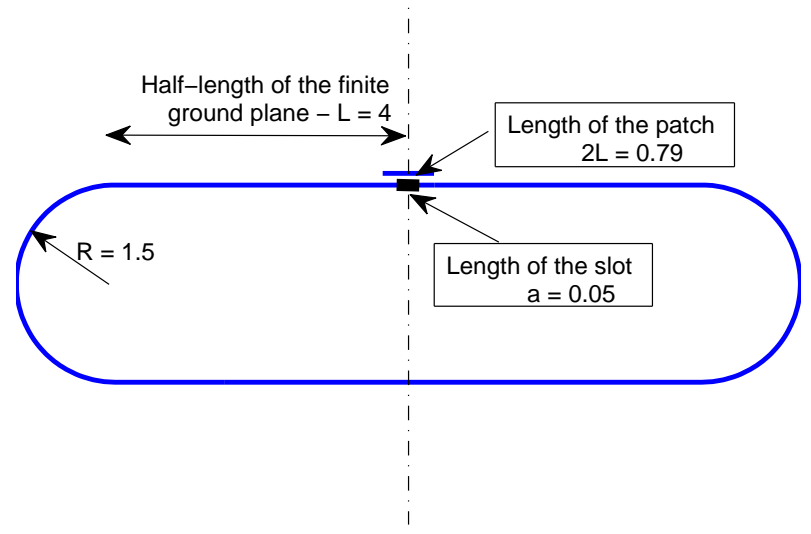

FIG. 4.1. Geometry of the configuration tested numerically.

\begin{tabular}{|c|c|c|c|c|c|c|}
\hline Dens & 60 & 50 & 40 & 30 & 20 & 10 \\
\hline DNS & 0.08 & 0.11 & 0.15 & 0.22 & 0.45 & 5.47 \\
Order 2 & 0.04 & 0.05 & 0.06 & 0.08 & 0.13 & 1.36 \\
Order 1 & 0.64 & 0.64 & 0.64 & 0.64 & 0.64 & 1.53 \\
Cavity & 1.10 & 1.10 & 1.10 & 1.12 & 1.23 & 2.58 \\
\hline
\end{tabular}

Errors in $d B$ for the four models requiring a numerical solution.

An exact analytical solution is not available for this problem. As a reference solution, we have solved the problem by using a DNS method on a very refined mesh built by taking dens $=260$. Table 4.1 gives the maximum error in $\mathrm{dB}$ over all directions $0 \leq \theta \leq 360^{\circ}$. It is computed for various meshes from very refined to standard and for the four models capable of yielding the radiation pattern in every direction. It turns out that the DNS model can lead to erroneous results when used with standard meshes of 10 nodes per wavelength. The results clearly confirm the validity of the asymptotic expansion approach as well as the improvement gained by pushing the expansion to the second order.

In Fig. 4.2 and Fig. 4.3, we depict the plots of the radiation patterns obtained for the more and the less refined meshes. For dens $=60$, the plots for the three models: DNS, 2nd- and 1st-order asymptotic expansion cannot be distinguished. It is worth mentioning that the erroneous results obtained when using a DNS method on standard meshes are limited to the backward directions.

The plots depicted in Fig. 4.2 and Fig. 4.3 clearly show that the radiation pattern obtained by means of the analytical formula (4.4) is sufficiently accurate in the appropriate directions $\left(30^{\circ} \leq \theta \leq 150^{\circ}\right)$ for the functioning of the antenna. Table 4.2 gives a comparison of all the models within this range of directions. 


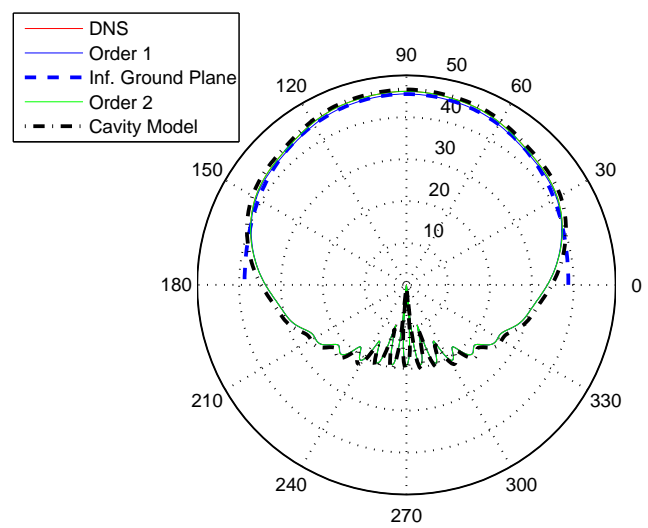

FIG. 4.2. Radiation patterns obtained by the various models on meshes of 60 points per wavelength.

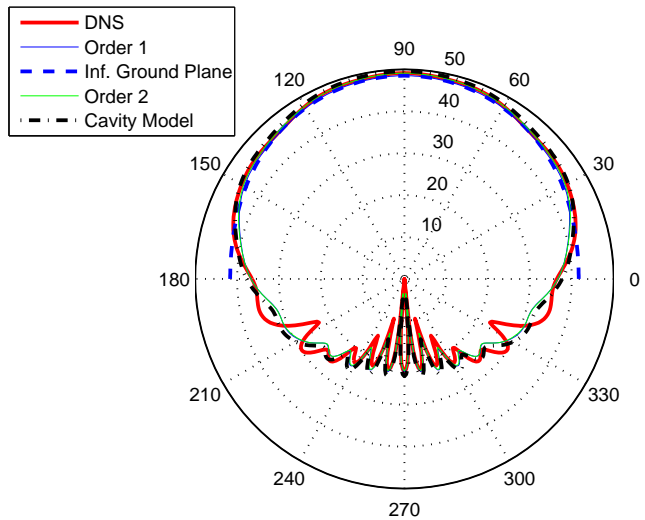

FIG. 4.3. Radiation patterns obtained by the various models on meshes of 10 points per wavelength.

Table 4.3 reports the CPU time in seconds used to carry out these numerical simulations. The CPU time used by the cavity model is the same as for the first-order asymptotic expansion since the two models only differ by the value for the parameter fixing the equivalent point sources: $\partial_{x} u_{\mathrm{c}}^{\mathrm{cm}}(L)$ and $\partial_{x} u_{\mathrm{c}}^{0,0}(L)$ (see (2.4) and (3.21) with (3.41)). This table also brings out the efficiency of the model corresponding to the second-order asymptotic expansion when it is solved using standard meshes.

The plot depicted in FIG. 4.4 accounts for the relative error in $\%$ on the value of the point source parameter $\partial_{x} u_{\mathrm{c}}^{\mathrm{cm}}(L)$ when it is approximated by more than one mode. Even if the error which results from the approximation of the exact value $\partial_{x} u_{\mathrm{c}}^{0,0}(L)$ by using only one term for the modal expansion is around $10 \%$, the discrepancy it yields on the determination of the radiation pattern is generally considered as 


\begin{tabular}{|c|c|c|c|c|c|c|c|}
\hline Dens & 60 & 50 & 40 & 30 & 20 & 10 & Meshless \\
\hline DNS & 0.01 & 0.01 & 0.02 & 0.02 & 0.05 & 0.30 & - \\
Order 2 & 0.03 & 0.02 & 0.03 & 0.02 & 0.04 & 0.16 & - \\
Order 1 & 0.64 & 0.64 & 0.64 & 0.64 & 0.64 & 0.66 & - \\
Cavity & 0.81 & 0.81 & 0.80 & 0.80 & 0.79 & 0.63 & - \\
Inf. Gr. & - & - & - & - & - & - & 0.90 \\
Plane & & \multicolumn{7}{|c|}{ TABLE 4.2} \\
\hline
\end{tabular}

Maximum error in $d B$ for $30^{\circ} \leq \theta \leq 150^{\circ}$.

\begin{tabular}{|c|c|c|c|c|c|c|c|c|}
\hline Dens & 260 & 60 & 50 & 40 & 30 & 20 & 10 & Meshless \\
\hline DNS & 83.01 & 3.14 & 2.19 & 1.38 & 0.83 & 0.45 & 0.17 & - \\
\hline Order 2 & - & 41.03 & 19.30 & 8.41 & 3.78 & 1.58 & 0.72 & - \\
\hline Order 1 & - & 2.19 & 1.56 & 1.05 & 0.73 & 0.38 & 0.31 & - \\
\hline $\begin{array}{c}\text { Inf. Gr. } \\
\text { Plane }\end{array}$ & - & - & - & - & - & - & - & $\begin{array}{c}\text { Too small } \\
\text { to be } \\
\text { estimated }\end{array}$ \\
\hline
\end{tabular}

CPU times for various models and discretizations.

acceptable when dealing with a real 3D problem. The easy way with which the radiation pattern is obtained in the appropriate directions for the functioning of the patch antenna largely compensates for the discrepancy resulting from this a priori crude approximation (see, for example, $[1,4]$ ).

5. Conclusion. Even limited to a $2 \mathrm{D}$ representation of the electromagnetic phenomenon, this study in our opinion provides a comprehensive basis for approaches which should be adopted in the numerical simulation of patch antennas. The presented theoretical and numerical investigations have brought out that a second-order model would be very useful for improving the numerical simulations of the functioning of such devices. Further studies are however necessary before the full 3D problem can be tackled.

Acknowledgement. The authors would like to acknowledge the thorough reading of the referees and to specially thank the comments of one of them which contribute to improve the final appearance of this paper.

\section{REFERENCES}

[1] C. A. Balanis, Antenna Theory: Analysis and Design, Third Edition, Wiley-Interscience, New-Jersey, 2005.

[2] A. Bendali and M. Fares, Boundary Integral Equations Methods in Acoustics, Saxe-Coburg Publications, f. Magoules ed., 2008, ch. 1, pp. 1-36.

[3] G. Caloz, M. Costabel, M. Dauge, and G. Vial, Asymptotic expansion of the solution of an interface problem in a polygonal domain with thin layer, Asymptotic Analysis, 50 (2006), pp. $121-173$.

[4] K. R. CARver And J. W. Mink, Microstrip Antenna Technology, IEEE Trans. Antennas Propagation, 29 (1981), pp. 2-24.

[5] D. Colton And R. KRess, Integral Equation Methods in Scattering Theory, John Wiley and Sons, New York, 1983. 


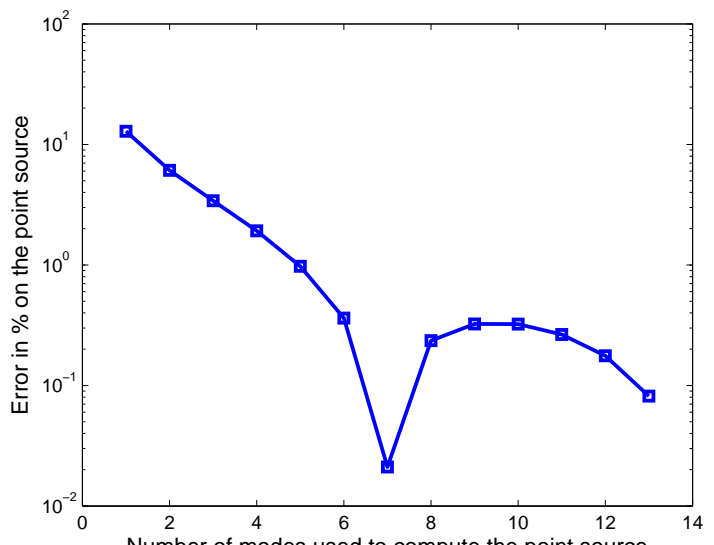

FIG. 4.4. Error on the point source parameter corresponding to the number of modes used for its approximation.

[6] G. C. Hsiao and W. L. Wendland, Boundary Iintegral Equations, Springer, BerlinHeidelberg, 2008.

[7] Jianming Jin, The Finite Element Method in Electromagnetics, Second Edition., John Wiley \& Sons, New York, 2002.

[8] P. Joly AND S. ToRdeux, Matching of Asymptotic Expansions for Wave Propagation in Media with Thin Slots i: The Asymptotic Expansion, Multiscale Modeling and Simulation: A SIAM Interdisciplinary Journal, 5 (2006), pp. 304-336.

[9] A. Makhlouf, Justification et Amélioration de Modèles d'Antennes Patch par la Méthode des Développements Asymptotiques Raccordés, PhD thesis, INSA Toulouse, 2008.

[10] P. MCIver AND A. D. RAWLINS, Two-dimensional wave-scattering problems involving parallelwalled ducts, Quart. J. Mech. Appl. Math., 46 (1993), pp. 89-116.

[11] J. R. Mosig and F. E. Gardiol, General Integral Equation Formulation for Microstrip Antennas and Scatterers, Proc. IEE, 132 (1985), pp. 424-432.

[12] J.-C. NÉDÉLEC, Acoustic and Electromagnetic Equations: Integral Representations for Harmonic Problems, Springer, Berlin, 2001.

[13] Andrew F. Peterson, Scott L. Ray, and Raj Mittra, Computational Methods for Electromagnetics, IEEE Press, Piscataway, New-Jersey, 1998.

[14] Z. X. Wang And D. R. Guo, Special Functions, World Scientific Publishing Co. Inc., Teaneck, New-Jersey, 1989. 